\title{
ENTIRE SOLUTIONS OF THE ABSTRACT CAUCHY PROBLEM IN A HILBERT SPACE
}

\author{
RALPH DELAUBENFELS AND FUYUAN YAO
}

(Communicated by Palle E. T. Jorgensen)

Abstract. We show that, whenever the linear operator $A$ is symmetric and densely defined, on a Hilbert space, then the abstract Cauchy problem

$$
\frac{d}{d z} u(z)=A^{*}(u(z)) \quad(z \in \mathbf{C}), \quad u(0)=x
$$

has an entire solution, for all initial data $x$ in the image of $e^{-\bar{A} A^{*}}$, which is a dense set.

\section{INTRODUCTION AND MAIN RESULTS}

Let $A$ be a densely defined linear operator on a Hilbert space $H$. A vector $x$ is an entire vector for $A$ if $x$ is in the domain of $A^{n}$, for all $n$, and

$$
\sum_{n=0}^{\infty} \frac{s^{n}}{n !}\left\|A^{n} x\right\|<\infty,
$$

for all $s>0$.

We will write $\mathscr{E}(A)$ for the set of all entire vectors for $A$.

It is clear that when $x \in \mathscr{E}(A)$, we have an entire solution of the abstract Cauchy problem

$$
\frac{d}{d t} u(t)=A(u(t)) \quad(t \geq 0), \quad u(0)=x,
$$

given by

$$
u(z) \equiv \sum_{n=0}^{\infty} \frac{z^{n}}{n !} A^{n} x \quad(z \in \mathbf{C}) .
$$

We also have well-posedness on the continuously embedded Frechet space $\mathscr{E}(A)$; that is, the restriction of $A$ to $\mathscr{E}(A)$ generates a strongly continuous semigroup (see [1], [4] or [6] for results about entire and analytic vectors).

When $A$ has a self-adjoint extension, entire solutions of the abstract Cauchy problem for all initial data in a dense set would follow from applying the spectral theorem to such an extension. We would like to consider $A$ to be an arbitrary

Received by the editors February 28, 1994 and, in revised form, April 7, 1994.

1991 Mathematics Subject Classification. Primary 47B25, 34G10, 47D05.

This work is part of the second author's Ph.D. Thesis at Ohio University. 
densely defined symmetric operator. On a larger Hilbert space, $K, A$ has a self-adjoint extension (see [7]), thus we automatically obtain entire solutions in $K$; we would like to have entire solutions in the original Hilbert space $H$.

In this paper, we show that, if $A$ is a densely defined linear symmetric operator on a Hilbert space, then $\mathscr{E}\left(A^{*}\right)$, where $A^{*}$ is the adjoint of $A$, contains the image of $e^{-\bar{A} A^{*}}$. Note that, since $\bar{A} A^{*}$ is a positive self-adjoint operator (see [7]), $\left\{e^{-t \bar{A} A^{*}}\right\}_{t \geq 0}$ is defined as a strongly continuous holomorphic semigroup generated by $-\bar{A} A^{*}$. Since the image of $e^{-\bar{A} A^{*}}$ is dense, this produces entire solutions of (ACP), with $A$ replaced by $A^{*}$, for all initial data in a dense set.

Theorem 1.1. Suppose $e^{i \theta} A$ is symmetric, for some real $\theta$. Then $\operatorname{Im}\left(e^{-\bar{A} A^{*}}\right) \subseteq$ $\mathscr{E}\left(A^{*}\right)$.

We also show, under the same hypotheses on $A$, that $\left[\begin{array}{cc}0 & 1 \\ A^{*} & 0\end{array}\right]$, on $H^{2}$, has a dense set of entire vectors. This produces entire solutions of the second-order abstract Cauchy problem

$$
\left(\frac{d}{d t}\right)^{2} u(t)=A(u(t)) \quad(t \geq 0), \quad u(0)=x, \quad \frac{d u}{d t}(0)=y,
$$

for all initial data $x, y$ in a dense set.

We write $I_{2}$ for the identity matrix in $H^{2}$.

Theorem 1.2. Suppose $e^{i \theta} A$ is symmetric, for some real $\theta$. Then $\operatorname{Im}\left(e^{-\bar{A} A^{*}} I_{2}\right) \subseteq$ $\mathscr{E}\left(\left[\begin{array}{cc}0 & 1 \\ A^{*} & 0\end{array}\right]\right)$.

The key to these results is the following.

Lemma 1.3. If $A$ is as in Theorems 1.1 and 1.2, then there exists a constant $M$ so that $e^{-\bar{A} A^{*}} x$ is in the domain of $\left(A^{*}\right)^{n}$, with

$$
\left\|\left(A^{*}\right)^{n} e^{-\bar{A} A^{*}} x\right\| \leq M^{n} n^{n / 2}\|x\|,
$$

for all $n \in \mathbf{N}, x \in H$.

Proof. Without loss of generality, we may assume $\theta=0$. Since the domain of $\bar{A} A^{*}, \mathscr{D}\left(\bar{A} A^{*}\right)$, is contained in $\mathscr{D}\left(\left(A^{*}\right)^{2}\right)$, it follows that $\operatorname{Im}\left(e^{-\bar{A} A^{*}}\right) \subseteq$ $C^{\infty}\left(\bar{A} A^{*}\right) \subseteq C^{\infty}\left(A^{*}\right) \equiv \bigcap_{n=0}^{\infty} \mathscr{D}\left(A^{n}\right)$. Since $\bar{A} A^{*}$ is self-adjoint and positive (see [7]), $-\bar{A} A^{*}$ generates a bounded holomorphic strongly continuous semigroup $\left\{e^{-t \bar{A} A^{*}}\right\}_{t \geq 0}$. This implies (see [3]) that there exists a constant $M$ so that

$$
\left\|\bar{A} A^{*} e^{-t \bar{A} A^{*}} x\right\| \leq \frac{M}{t}\|x\|, \quad \forall x \in H, t>0 .
$$

By (1.5),

$$
\left\|\left(A^{*}\right)^{2 n} e^{-\bar{A} A^{*}} x\right\|=\left\|\left(\bar{A} A^{*}\right)^{n} e^{-\bar{A} A^{*}} x\right\|=\left\|\left(\bar{A} A^{*} e^{-\frac{\bar{A} A^{*}}{n}}\right)^{n} x\right\| \leq M^{n} n^{n}\|x\|,
$$

for all $x \in H, n \in \mathbf{N}$. Assertion (1.6) now implies that

$$
\begin{aligned}
\left\|\left(A^{*}\right)^{2 n+1} e^{-\bar{A} A^{*}} x\right\|^{2} & =\left\langle\left(A^{*}\right)^{2 n+1} e^{-\bar{A} A^{*}} x,\left(A^{*}\right)^{2 n+1} e^{-\bar{A} A^{*}} x\right\rangle \\
& =\left\langle\bar{A}\left(A^{*}\right)^{2 n+1} e^{-\bar{A} A^{*}} x,\left(A^{*}\right)^{2 n} e^{-\bar{A} A^{*}} x\right\rangle \\
& =\left\langle\left(A^{*}\right)^{2 n+2} e^{-\bar{A} A^{*}} x,\left(A^{*}\right)^{2 n} e^{-\bar{A} A^{*}} x\right\rangle \\
& \leq M^{2 n+1}(n+1)^{n+1} n^{n}\|x\|^{2},
\end{aligned}
$$


so that

$$
\left\|\left(A^{*}\right)^{2 n+1} e^{-\bar{A} A^{*}} x\right\| \leq M^{n+1}(n+1)^{n+\frac{1}{2}}\|x\|, \quad \forall x \in H, n \in \mathbf{N} .
$$

Clearly (1.6) and (1.7) imply (1.4).

Proof of Theorem 1.1. This follows from Lemma 1.3 and a short, elementary calculation.

Proof of Theorem 1.2. This follows from Lemma 1.3 and the fact that

$$
\begin{aligned}
\sum_{n=0}^{\infty} \frac{s^{n}}{n !}\left[\begin{array}{cc}
0 & I \\
A^{*} & 0
\end{array}\right]^{n} e^{-\bar{A} A^{*}} & \\
= & {\left[\begin{array}{cc}
\sum_{n=0}^{\infty} \frac{s^{2 n}}{(2 n) !}\left(A^{*}\right)^{n} e^{-\bar{A} A^{*}} & \sum_{n=0}^{\infty} \frac{s^{2 n+1}}{(2 n+1) !}\left(A^{*}\right)^{n} e^{-\bar{A} A^{*}} \\
\sum_{n=0}^{\infty} \frac{s^{2 n+1}}{(2 n+1) !}\left(A^{*}\right)^{n+1} e^{-\bar{A} A^{*}} & \sum_{n=0}^{\infty} \frac{s^{2 n}}{(2 n) !}\left(A^{*}\right)^{n} e^{-\bar{A} A^{*}}
\end{array}\right], }
\end{aligned}
$$

for any $s>0$.

Remarks 1.8. (a) When $A$ is a symmetric differential operator, with $\bar{A} \neq A^{*}$, then, intuitively, $A^{*}$ has "too many boundary conditions" restricting its domain. Passing to $A^{*}$, as in Theorems 1.1 and 1.2 , has the effect of removing some, and sometimes all, boundary conditions, while still guaranteeing entire solutions of the corresponding abstract Cauchy problem, for all initial data in a dense set. This is of particular interest on a bounded domain, where boundary conditions may become delicate.

(b) We remark that it is clear that we obtain entire solutions of (ACP), with $A$ replaced by $A^{*}$, when we choose initial data $x$ in a deficiency subspace of $A^{*}$, since these are eigenvectors of $A^{*}$. However, the span of these subspaces may not be dense.

(c) Here is another way of viewing Theorem 1.1. In searching for a selfadjoint operator, one must verify two conditions:

(1) a numerical range condition $\langle A x, x\rangle \in \mathbf{R}$, for all $x$ in the domain of $A$ (this is equivalent to $A$ being symmetric) and

(2) a range condition ( $i \pm A$ ) being surjective.

In practice, verifying (2) is much more difficult than verifying (1). Our result removes the necessity of verifying (2).

Remark 1.9. Theorems 1.1 and 1.2 may be understood most easily in the context of existence families; these are a generalization of strongly continuous semigroups, that are meant to play the same role with ill-posed abstract Cauchy problems that strongly continuous semigroups play with well-posed abstract Cauchy problems. Theorem 1.1 is stating that $A^{*}$ has an entire $e^{-\bar{A} A^{*}}$-existence family, which we think of as the family of bounded operators $\left\{e^{z A^{*}} e^{-\bar{A} A^{*}}\right\}_{z \in \mathrm{C}}$, just as we think of a strongly continuous semigroup generated by $B$ as $\left\{e^{t B}\right\}_{t \geq 0}$. See [2] for an introduction to the subject, and some examples and applications.

\section{TWo ILlustrative EXAMPLES}

Example 2.1. The following example, although quite simple, is nevertheless sufficient to illustrate some interesting features of Theorem 1.1. 
Let $A \equiv i \frac{d}{d x}$, on $H \equiv L^{2}([0, \infty))$, with domain $\mathscr{D}(A) \equiv\left\{f \in H \mid f^{\prime} \in\right.$ $H, f(0)=0\}$. Then $A$ is symmetric, but (ACP), with $A$ replaced by $i A$, has no nontrivial reversible solutions, that is,

$$
\frac{d}{d t} u(t)=i A(u(t)) \quad(t \in \mathbf{R}), \quad u(0)=f
$$

has no nontrivial solutions.

Theorem 1.1 guarantees that

$$
\frac{d}{d t} u(t)=i A^{*}(u(t)) \quad(t \in \mathbf{R}), \quad u(0)=f
$$

has an entire solution, for all initial data $f$ in a dense set. This is merely saying that there exists a dense set of functions $f$, each of which has an entire extension, $\tilde{f}$, such that $[u(z)](s) \equiv \tilde{f}(s+z)$ is in $H$, for all $z \in \mathbf{C}$.

For any $f \in H,(2.3)$ has a mild solution. However, this solution is not unique. For any $f \in L^{2}([0, \infty))$, choose any $\tilde{f} \in L^{2}(\mathbf{R})$ that extends $f$. Then

$$
[u(t)](s) \equiv \tilde{f}(s+t) \quad(s \geq 0, t \in \mathbf{R})
$$

is a mild solution of $(2.3)$.

It is clear that an entire solution of (2.3), or, more generally, (ACP), is unique.

The motivation for Theorem 1.1 is clear. If $(A f)(w) \equiv w f(w)$, on $H \equiv$ $L^{2}(\mathbf{C}, \mu)$, for some Borel measure $\mu$, on the complex plane $\mathbf{C}$, then it is straightforward to verify that

$$
(u(z))(w) \equiv e^{z w} e^{-|w|^{2}} f(w)
$$

is an entire solution of (ACP), with initial data $x(w) \equiv e^{-|w|^{2}} f(w)$, for any $f \in H$. The adjoint of an operator is a generalization of complex conjugation; thus a natural conjecture is that, for any densely defined linear operator $A$, either $\mathscr{E}(A)$ or $\mathscr{E}\left(A^{*}\right)$ contains the image of $e^{-\bar{A} A^{*}}$. The following example shows that this conjecture is false.

Counterexample 2.4. This is an example of a densely defined operator, $A$, on a Hilbert space, such that neither $\mathscr{E}(A)$ nor $\mathscr{E}\left(A^{*}\right)$ contains the image of $e^{-\bar{A} A^{*}}$.

Let $\mathrm{H}$ be $l_{2}(Z)$, the space of square summable sequences

$$
\left\{\left.\vec{x}\left|\|\vec{x}\|^{2} \equiv \sum_{k=-\infty}^{\infty}\right| x_{k}\right|^{2}<\infty\right\} .
$$

For any integer $k$, write $\vec{e}_{k}$ for the sequence whose $k$ th coordinate is one, all other coordinates are zero.

Let $\left\{\omega_{k}\right\}_{k \in Z}$ be a fixed sequence of nonnegative numbers. Define $A$ to be the weighted left-shift

$$
A \vec{e}_{k} \equiv \omega_{k} \vec{e}_{k-1} \quad(k \in Z),
$$

with maximal domain $\mathscr{D}(A) \equiv\{\vec{x} \in H \mid A \vec{x} \in H\}$.

Then $A^{*}$ is the weighted right-shift

$$
A^{*} \vec{e}_{k} \equiv \omega_{k+1} \vec{e}_{k+1} \quad(k \in Z),
$$

and $A A^{*}$ is the positive operator

$$
A A^{*} \vec{e}_{k} \equiv\left|\omega_{k+1}\right|^{2} \vec{e}_{k} \quad(k \in Z) ;
$$


note that $-A A^{*}$ generates the strongly continuous semigroup

$$
e^{-t A A^{*}} \vec{e}_{k} \equiv e^{-t\left|\omega_{k+1}\right|^{2}} \vec{e}_{k} \quad(t \geq 0, k \in Z)
$$

It is not hard to calculate that, for any $n$,

$$
\begin{aligned}
A^{n} e^{-\bar{A} A^{*}} \vec{e}_{0} & =e^{-\left|\omega_{1}\right|^{2}}\left(\prod_{j=0}^{n-1} \omega_{-j}\right) \vec{e}_{-n}, \\
\left(A^{*}\right)^{n} e^{-\bar{A} A^{*}} \vec{e}_{0} & =e^{-\left|\omega_{1}\right|^{2}}\left(\prod_{j=1}^{n} \omega_{j}\right) \vec{e}_{n} .
\end{aligned}
$$

Thus, by choosing $\left\{\omega_{k}\right\}_{k \in Z}$ sufficiently large, so that

$$
\sum_{n=0}^{\infty} \frac{s^{n}}{n !}\left(\prod_{j=1}^{n} \omega_{ \pm j}\right)=\infty, \quad \text { for all } s>0
$$

(for example, $\omega_{k} \equiv 2|k|$, for $k \in Z$ ), we see that neither $\mathscr{E}(A)$ nor $\mathscr{E}\left(A^{*}\right)$ will contain $e^{-\bar{A} A^{*}} \vec{e}_{0}$.

\section{Applications}

We will apply Theorems 1.1 and 1.2 to differential operators in $L^{2}\left(\mathbf{R}^{n}\right)$. Suppose that, for $1 \leq i, j \leq n, a_{i, j}$ and $a_{i, j}^{\prime}$ are real-valued functions in $L_{\text {loc }}^{\infty}\left(\mathbf{R}^{n}\right)$, and for each $x \in \mathbf{R}^{n}, a_{i, j}(x)=a_{j, i}(x)$. Define $A$ on $L^{2}\left(\mathbf{R}^{n}\right)$ by

$$
(A f)(x) \equiv \sum_{i, j=1}^{n} \frac{\partial}{\partial x_{i}}\left(a_{i, j}(x) \frac{\partial f}{\partial x_{j}}(x)\right)
$$

with the domain of $A$ equal to $C_{c}^{\infty}\left(\mathbf{R}^{n}\right)$. Then $A$ is symmetric, and

$$
\left(A^{*} f\right)(x)=\sum_{i, j=1}^{n} \frac{\partial}{\partial x_{i}}\left(a_{i, j}(x) \frac{\partial f}{\partial x_{j}}(x)\right)
$$

with the domain of $A^{*}$ equal to $\left\{f \in L^{2}\left(\mathbf{R}^{n}\right) \mid A^{*} f \in L^{2}\left(\mathbf{R}^{n}\right)\right\}$.

Thus we may apply Theorems 1.1 and 1.2. It is clear that this produces entire (as a function of $t$ ) solutions of

$$
\frac{\partial u}{\partial t}(t, x)=\sum_{i, j=1}^{n} \frac{\partial}{\partial x_{i}}\left(a_{i, j}(x) \frac{\partial}{\partial x_{j}}\right) u(t, x), \quad u(0, x)=f(x)
$$

and

$$
\begin{aligned}
\frac{\partial^{2} u}{\partial t}(t, x) & =\sum_{i, j=1}^{n} \frac{\partial}{\partial x_{i}}\left(a_{i, j}(x) \frac{\partial}{\partial x_{j}}\right) u(t, x), \\
u(0, x) & =f(x), \quad \frac{\partial u}{\partial t}(0, x)=g(x),
\end{aligned}
$$

for all $f, g$ in a dense subset of $L^{2}\left(\mathbf{R}^{n}\right)$, with well-posedness on a dense, continuously embedded subspace.

As far as we know, this is the first treatment of so general a class of operators; this class includes nonelliptic operators. See [4, pp. 274-275] for some hypotheses when $n$ equals one that guarantee that $A$ has a self-adjoint extension. 


\section{REFERENCES}

1. L. Autret, Entire vectors and time reversible Cauchy problems, Semigroup Forum 46 (1993), 347-351.

2. R. deLaubenfels, Existence families, functional calculi and evolution equations, Lecture Notes in Math., vol. 1570, Springer-Verlag, New York, 1994.

3. J. A. Goldstein, Semigroups of operators and applications, Oxford Univ. Press, New York, 1985.

4. R. Goodman, Complex Fourier analysis on a nilpotent Lie group, Trans. Amer. Math. Soc. 160 (1971), 373-391.

5. T. Kato, Perturbation theory for linear operators, Springer-Verlag, New York, 1966.

6. E. Nelson, Analytic vectors, Ann. of Math. (2) 70 (1959), 572-615.

7. F. Riesz and B. Sz.-Nagy, Functional analysis, Ungar, New York, 1955.

Department of Mathematics, Ohio University, Athens, Ohio 45701

E-mail address: 72260.2403@COMPUSERVE. COM 